\title{
Surface-patterned micromechanical elements by polymer injection molding with hybrid molds
}

\author{
Helmut Schift ${ }^{\mathrm{a})}$ \\ Laboratory for Micro- and Nanotechnology (LMN), Paul Scherrer Institut (PSI), 5232 Villigen PSI, \\ Switzerland \\ Prabitha Urwyler \\ ARTORG Center for Biomedical Engineering Research, University of Bern, Murtenstrasse 50, \\ 3010 Bern, Switzerland \\ Per Magnus Kristiansen \\ Institute of Polymer Nanotechnology (INKA), University of Applied Sciences and Arts Northwestern \\ Switzerland (FHNW), 5210 Windisch, Switzerland
}

(Received 21 June 2013; accepted 19 August 2013; published 18 September 2013)

\begin{abstract}
Hybrid molds enable the fabrication of polymeric parts with features of different length scales by injection molding. The resulting polymer microelements combine optical or biological functionalities with designed mechanical properties. Two applications are chosen for illustration of this concept: As a first example, microelements for optical communication via fiber-to-fiber coupling are manufactured by combining two molds to a small mold insert. Both molds are fabricated using lithography and electroplating. As a second example, microcantilevers $(\mu \mathrm{Cs})$ for chemical sensing are surface patterned using a modular mold composed of a laser-machined cavity defining the geometry of the $\mu \mathrm{Cs}$, and an opposite flat tool side which is covered by a patterned polymer foil. Injection molding results in an array of $35 \mu \mathrm{m}$-thick $\mu \mathrm{Cs}$ with microscale surface topographies. In both cases, when the mold is assembled and closed, reliefs are transferred onto one surface of the molded element whose outlines are defined by the micromold cavity. The main advantage of these hybrid methods lies in the simple integration of optical surface structures and gratings onto the surface of microcomponents with different sizes and orientations. This allows for independent development of functional properties and combinations thereof. ( 2013 American Vacuum Society. [http://dx.doi.org/10.1116/1.4821649]
\end{abstract}

\section{INTRODUCTION}

Micromechanical elements, which are parts of microelectromechanical systems, can exhibit combinations of mechanical properties with optical or biological functionalities. Typical examples of these are microcantilevers $(\mu \mathrm{Cs})$ used as mechanical transducers in biological sensing platforms, in which the surface of the silicon $\mu \mathrm{C}$ is chemically modified to enable the adhesion and assembly of molecules. ${ }^{1,2}$ This changes either the mass or the stress on the cantilever, leading to static deflection or variation of dynamic (oscillatory or damping) properties. Readout of these changes can be accomplished by detecting a laser beam reflected off the cantilever surface, similar to the readout in atomic force microscopes. In this way, deflections due to bending are accurately monitored. Typical examples of micromechanical elements are gear wheels and springs for watches, membranes for pressure sensors, mirrors or adjustable lenses for light manipulation, as well as springs and holders for optical fiber interconnectors. ${ }^{3,4}$ In most cases, the mechanical property is determined by its three-dimensional (3D) geometry. In other words, the overall mechanical properties of the $\mu \mathrm{C}$ is given by the shape and dimensions of the cantilever beam as defined by its length, thickness, and the Young's modulus of the material from which it is made. ${ }^{5}$ In the case of a

a)Electronic mail: helmut.schift@psi.ch microgear, the height of its teeth determines the length of its contact line. Additional functionalities can be integrated onto the surface of these elements. For instance, Mimotec SA (Switzerland) fabricates metallic microgears with integrated holograms on its flat front side, which serve as anticounterfeit features. ${ }^{6}$ In both the $\mu \mathrm{C}$ and the microgear structure, the surface topography of the additional functionalization does not interfere with the main mechanical property of the structure, and has no or minimal effect upon its Young's modulus or the resonance frequency. In the case of the microgear, this is true because the teeth and, therefore, the mechanical behavior are only formed by the vertical sidewalls. In the case of $\mu \mathrm{Cs}$, the mechanical property depends very much on the depth, shape, and orientation of the functional structures on the $\mu \mathrm{C}$ beam. For example, a surface topography enhances the overall surface for biomolecule attachment and, thus, the possible added mass of a monolayer; the shape of structures can enhance the possibility of cell attachment; the orientation of a line pattern can determine in which direction they are able to exert stress or in which direction the cantilever beam bends. ${ }^{7}$ In any case, the mechanical properties of the beam will only change significantly if the depth of the surface corrugation compares with the cantilever thickness. ${ }^{8}$

Although for both, silicon $\mu \mathrm{Cs}$ and metal microgears, advanced batch-based microfabrication methods can be used, i.e., processes based on lithography and either silicon 
micromachining or electroplating, more cost effective methods need to be employed if true mass fabrication is required. Polymer replication technologies such as hot embossing (also known as thermal imprint) and injection molding allow straightforward mass production. Injection molding is established in industry and has been adapted to microinjection molding $(\mu \mathrm{IM})$ with structures down to $10 \mathrm{~nm} .{ }^{9,10}$ For metal microgears, it is already used as the third step of the established process sequence of the LiGA technology. A German acronym for lithography $(\mathrm{Li}=$ Lithographie), electroforming ( $\mathrm{G}=$ Galvanik), and molding ( $\mathrm{A}=$ Abformung), LiGA, is particularly suited for high aspect ratio microstructures (named HAR or HARMST), with patterning abilities to create precise vertical sidewalls and structures with aspect ratios of 20-100 and even greater. ${ }^{3,4}$ Another advantage of LiGA is the low surface roughness (down to a few nanometers) possible with the technique, which in the case of microgears is important even if this roughness does not distort the overall shape of the microgears. With the LiGA process, therefore, instead of fabricating metal microgears in batch processes directly, molds are fabricated. These molds consist of a metal frame surrounding a cavity possessing the desired geometrical outlines, which are inserted into standard injection molding machines and subsequently produce replicated parts. For entirely polymeric parts, the aim is to fabricate a mold which can be incorporated into the already existing locations of the respective tool sides (the clamping unit, opposite to the mirror unit at the injection side), which form a closed cavity with a specific volume when pressed together. The molten polymer (typical melt viscosities in the order of $100-1000 \mathrm{~Pa} \cdot \mathrm{s}$ ) is injected into the mold cavity through one or multiple inlets and allowed to cool down under applied pressure. Once solidified, the cavity is opened, and the part is demolded by separation of the tool halves and ejection of the molded part from the mold insert. For the applications described here, the mold needs to have welldefined topographies (e.g., gratings) on one or more of its surfaces that will be replicated during the $\mu \mathrm{IM}$, yielding a polymer part with precise surface structuring. Since we are mostly interested in adding the surface functionality locally without modifying the overall shape and performance of the element, the approach of hybrid molds is particularly interesting. In hybrid molds, the additional surface functionality is either added to an existing mold (e.g., by attaching a thin film onto it) or achieved by assembling different mold parts in an aligned way into a multicomponent mold insert. Alternatively, structured mold inserts are incorporated on opposite sides of the mold, whereby a truly hybrid mold is created only when the mold is closed during the injection cycle.

The use of $\mu \mathrm{IM}$ for manufacturing $\mu \mathrm{Cs}$ has already been reported. ${ }^{11}$ In contrast to the microgears, $\mu \mathrm{Cs}$ are more difficult to mold, mainly because of the smaller dimensions of the $\mu \mathrm{C}$ beams. In the following work, two examples of micromechanical elements with hybrid mold concepts are successfully applied to facilitate replication of microelements in a versatile manner during the $\mu \mathrm{IM}$ process. These examples are accomplished using a combined mold to produce fiber connectors following the LiGA approach described above, and with a modular mold to produce $\mu \mathrm{Cs}$ where two molds are attached to opposite units of the molding tool. Since the concepts behind both applications have been published elsewhere, ${ }^{12-15}$ this work focuses on specific new aspects relevant to these hybrid techniques.

\section{EXPERIMENT}

For $\mu \mathrm{IM}$, molds made from metal are generally preferred due to their durability. However, methods for structuring metallic molds are still limited to features in the micrometer range and nanostructures cannot yet be machined into tooling steel in a cost-effective manner. For mold variants, often small inserts are fabricated and placed into milled cavities of the mold. Such cavities can also be further processed by precision milling, e.g. for $\mu \mathrm{Cs}$ of different thickness. Different methods have been developed to shape surfaces with microscale precision and create cavity volumes of a few cubic millimeters. Some examples of these methods are electromachining with fine electrodes, intense pico- or femtosecond pulsed laser ablation and high-precision milling using diamond tools. In all cases, structural details down to a few micrometers have been achieved, though with different boundary conditions and restrictions in shape, roughness, and precision.

\section{A. Combined metal mold for the fabrication of micromechanical clamping elements}

Often microgears of different diameters are stacked together on one axis in order to provide for power transmission. Stacked elements can be fabricated simply by using iterations of the lithography and electroplating steps of the LiGA sequence. Another possibility is to combine several individual mold parts as a stack to create an integer mold insert. For this option, the individual mold frames are fabricated using the well-established LiGA process, by starting with a maskbased photolithography using either deep x-rays or UV light. During wet development, part of the resist structure is dissolved down to the conductive substrate surface. The trenches between the remaining HAR resist structures with straight vertical sidewalls are filled with metal by electroplating, typically with nickel or nickel alloys, followed by resist removal. The resulting metallic structures are released from the plating base and are then used as mold inserts with defined height, being incorporated into the molding tool by clamping or sometimes even gluing. Small microelements confined by the outlines of a frame and the front and back surface of the cavity are then replicated in the $\mu \mathrm{IM}$ process. If one or both surfaces contain a defined surface topography, the molded polymer element will feature both the designed geometry as outlined by the frame and the desired functional topography on different surfaces with defined geometrical reference.

A good example of a combined structure of this type is a fiber interconnect element with diffractive optical elements (DOEs) on the front surface and with mechanical registration given by the straight sidewalls. This is particularly useful for the coupling of light from optical fibers into integrated optics or for the manipulation of light transmission between entire 
fiber bundles. The mechanical registration is needed because often such fibers are placed in special holders with "rails" or pins provided by a fiber connector or an optical microbench. All fibers and lenses must be centered with respect to the optical axis of the optical beam, which requires that the lenses be aligned within a defined frame that fits into the registration of the rails. These lenses are therefore placed at the bottom of the LiGA mold, situating them at the area of the substrate from which the $500 \mu \mathrm{m}$ high metal HAR structures are grown (see Figs. 1 and 2). The details of this process are presented in Ref. 13. For flexibility, in this work, it was easier to fabricate a separate mold than to integrate the lenses into the plating base of the LiGA mold. These lens elements were fabricated in a process sequence similar to LiGA, but starting with a grayscale-type optical lithography of a few micrometers high structures from which a Ni shim of $200 \mu \mathrm{m}$ thickness was electroplated. The DOEs were designed to enable focusing and collimation of light from semiconductor lasers or single-mode fibers. Results of fiberto-fiber coupling achieved with hot embossing are presented in Ref. 12. Owing to a lateral size of the mold insert of $7 \times 7 \mathrm{~mm}^{2}$ (to make effective use of the LiGA lithography mask), smaller cavities of $5 \times 3 \mathrm{~mm}^{2}$ were achieved by clamping with four screws on a $7 \times 7 \times 6.3 \mathrm{~mm}^{3}$ aluminum cube with M1.8 screw holes (see Fig. 2). For larger cavities of $5 \times 5 \mathrm{~mm}^{2}$, the two metal elements were point soldered using a $0.5 \mathrm{~mm}$ diameter metal tip. Proper alignment was achieved under an optical microscope with the help of a special displacement setup, which allowed the LiGA frame to be moved with respect to the lens shim with submicrometer precision and subsequent clamping by slight pressure. Opposite, on the mirror unit, a polished steel insert was placed which constitutes of the flat optical back of the optical microelements. Furthermore, this mirror unit enables injection of the melted polymer with up to 16 inlets of $500 \mu \mathrm{m}$ diameter. In addition to the molding elements

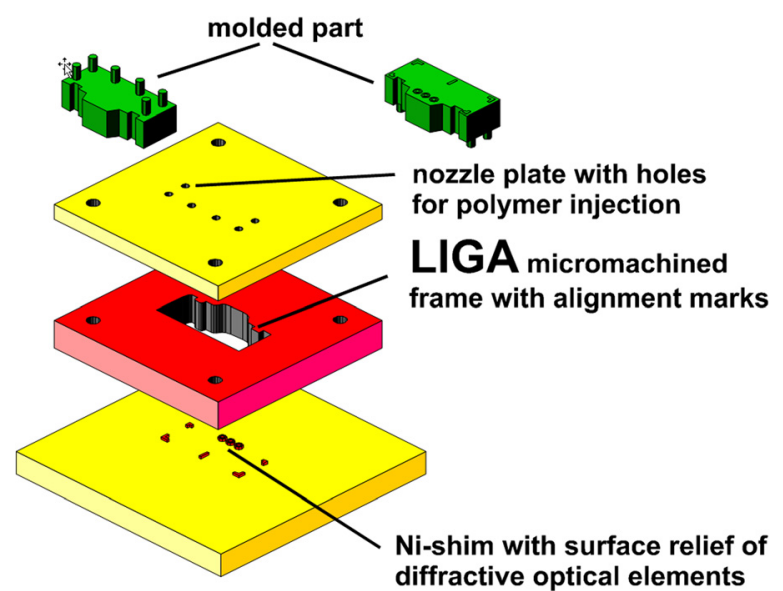

FIG. 1. (Color online) Combined mold insert with a $500 \mu$ m-high LiGA frame, a bottom plate with diffractive optical elements placed at the clamping unit of the injection molding tool, and a top plate with inlets for polymer melt placed at the mirror unit. The mold separation plane is between the nozzle plate and the LiGA frame. Reproduced with permission from Söchtig et al., Proc. SPIE 3226, 44 (1997); Fig. 7. Copyright 1997, SPIE-The International Society for Optical Engineering.

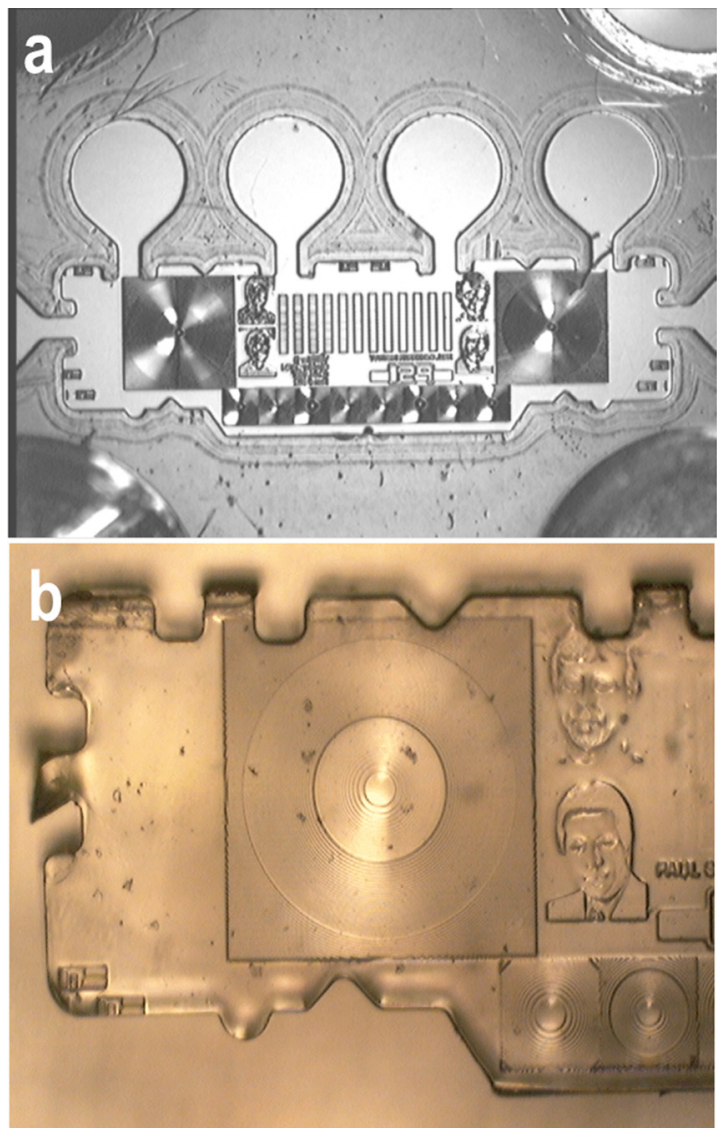

FIG. 2. (Color online) Microelements designed for insertion into a silicon miocrobench with etched rails for registration. (a) Mold with outlines defined by the LiGA frame and by the DOE-lenses in the center. The four orifices at the upper side represent auxiliary structures for injection and pullout during demolding. (b) Injection molded micro-optical element in polycarbonate with lenses at the front side. Reproduced with permission from Schift et al., Proc. SPIE 3513, 122 (1998); Figs. 4 and 7. Copyright 1998, SPIE-The International Society for Optical Engineering.

presented in Ref. 13, which were designed for the insertion into micromachined microbenches in silicon, we present here a specific element which was intended to be used as an optical interconnect element between two fiber connectors (so-called MT connector for ribbon cables), which were designed to align two fiber bundles with eight fibers each (see Fig. 3). The lenses of the fibers are aligned with respect to a MT fiber connector by clamps sitting on $700 \mu \mathrm{m}$ steel rods. The lever with a thickness of about $200 \mu \mathrm{m}$ and a length of $1000 \mu \mathrm{m}$ was designed to be long enough to enable snapping of the elements on the fiber rods through springs, and to be strong enough to enable a tight fitting of the rods. The injection takes place both through orifices at the bulk of the microelements as well as through the tips of each lever constituting the clamps, which was considered more advantageous than a single central injection point. These orifices also serve as mechanical pillars to pull the element with its complex outlines out of the mold during demolding. Different materials were used during variothermal $\mu \mathrm{IM}$, including optical-grade poly methyl methacrylate and polycarbonate. Figure 3 shows a collimation arrangement for fiber-to-fiber coupling using the injection molded microelements (shown here for demonstration only). Two 


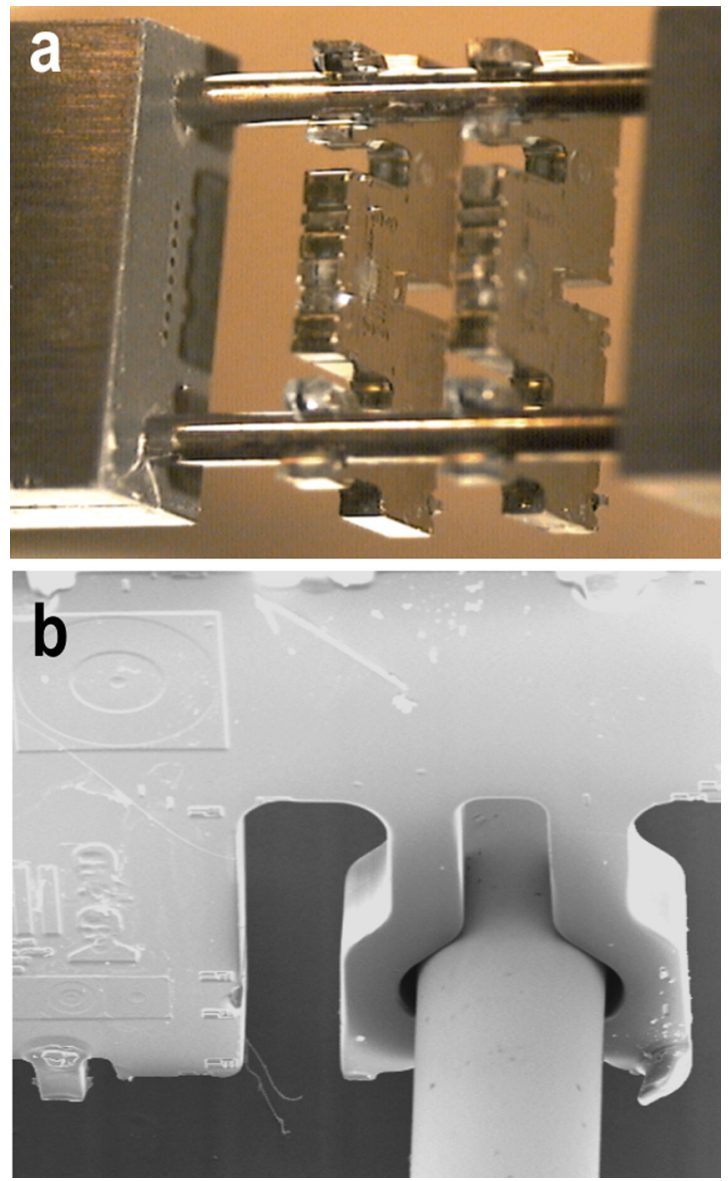

FIG. 3. (Color online) Collimation arrangement for fiber-to-fiber coupling using injection molded microelements. (a) Two micro-optical elements in polycarbonate are clamped on MT-connector guide pins $(700 \mu \mathrm{m})$. (b) The lenses at the front side of the elements are designed to collimate the light of eight fibers.

polycarbonate micro-optical elements are clamped on the MT-connector guide pins. The lenses at the front side of the elements are designed to collimate the light from singlemode fibers.

\section{B. Modular mold with polymer foil insert for the fabrication of surface-patterned microcantilevers}

An example of a modular mold in which the microcavity and surface pattern mold are attached to two opposite sides of a $\mu \mathrm{IM}$ tool is one that fabricates $\mu \mathrm{Cs}$ with a defined surface topography. The $\mu \mathrm{Cs}$ may serve to measure a cell force if their surface is patterned with linear gratings suitable for cell attachment. ${ }^{7}$ The same overall outline as the one used for a silicon $\mu \mathrm{C}$ array (eight $\mu \mathrm{Cs}$ with $500 \times 80 \mu \mathrm{m}^{2}$ lateral size and $250 \mu \mathrm{m}$ pitch) were chosen, which are connected to a larger body of $2 \times 3 \times 0.5 \mathrm{~mm}^{3}$. Microcantilevers respond to impact resulting from a wide range of surface stresses, induced by changes, for example, in mass or temperature, through the ability to detect minute changes in the deflection or resonance frequency of the $\mu \mathrm{C}$. Forces in the pN-range are detectable, since the setup can measure subnanometer deflections of the apex of the $\mu \mathrm{C}$ sensors. These forces are due to expansions or contractions acting on one side of the cantilever surface. The application of static and dynamic modes can add sophistication to the sensing and measurements. In static mode, the surface stress generated from selectively adsorbed molecules on one side of the cantilever is measured. The free-end deflection, $\Delta_{\mathrm{z}}$, which occurs as the result of the surface stress, $\sigma_{\text {surface}}$, is often quantified using the well-known Stoney formula. The Sader form of the Stoney formula is given as ${ }^{5}$

$$
\Delta_{z}=\frac{3(1-\nu) L^{2}}{E t^{2}} \cdot \Delta \sigma_{\text {surface }}
$$

where $\Delta \sigma_{\text {surface }}$ is the difference in surface stress between top and bottom sides of the cantilever, $\nu$ is Poisson's ratio, $E$ is the Young's modulus of the cantilever material, and $L$ and $t$ are the length and thickness of the cantilever, respectively.

The ramifications of the Stoney formula for this design means that, in order to have a sensitivity similar to a silicon $\mu \mathrm{C}$, a polymer $\mu \mathrm{C}$ needs to be about 7 times thicker. For sensitivities equivalent to $1 \mu \mathrm{m}$-thick $\mathrm{Si}$ cantilevers, this would mean a polymeric thickness of $7 \mu \mathrm{m}$, which is far thinner than the current capabilities of $\mu \mathrm{IM}$ processes, since mold gaps below $10 \mu \mathrm{m}$ or even larger are considered "closed." To facilitate both the mold fabrication and replication, thicker $\mu \mathrm{Cs}$ in the range between 25 and $50 \mu \mathrm{m}$ were chosen. The cavities were laser ablated in PolMax (Polmax Uddeholm) steel with an overall roughness below $1 \mu \mathrm{m}$, and $10 \times 10 \mu \mathrm{m}^{2}$ venting channels were introduced at the tip of the $\mu \mathrm{C}$ cavities to allow displacement of air during injection. For polypropylene (PP: Metocene HM648T LyondellBasell, Bayreuth, Germany), $500 \times 80 \times 45 \mu \mathrm{m}^{3}$ large $\mu \mathrm{C}$ beams could be completely filled using isothermal molding conditions, whereas only incomplete filling was achieved with $35 \mu \mathrm{m}$ thick $\mu \mathrm{C}$ beams. A polymer foil was placed on the mirror unit (i.e., the unstructured flat side of the molding tool) from which also the injection took place through a large injection gate (see Fig. 4). This was done for two reasons: (1) Polymer foils drastically reduce the heat loss from the melt into the mold cavity walls caused by the high thermal conductivity of typical mold materials, such as $\mathrm{Ni}$ or steel. Because of the preserved heat in the cantilever area, the melt temperature remains above its solidification temperature until the entire $\mu \mathrm{C}$ beam cavity (with an "aspect ratio" of 13) is filled. ${ }^{16-18}$ (2) Patterning of polymer foils by hot embossing is a versatile means by which surface topographies can be created in the form of exchangeable auxiliary mold inserts. Transfer of these topographies onto the surface of the $\mu \mathrm{C}$ can be accomplished without any changes in the molding process. Moreover, these foils did not require any alignment because only large area gratings with surface areas larger than $10 \times 10 \mathrm{~mm}^{2}$ were used. An alignment of these foils using an improvised fixation setup using Scotch tape and the alignment of the two tool units was roughly specified to be in the mm-range. For the foil, amorphous polyetheretherketone (PEEK) was used (APTIV 2000, $25 \mu \mathrm{m}$ ), which could be patterned using hot embossing at about $220^{\circ} \mathrm{C}$, and was also able to withstand the $200^{\circ} \mathrm{C}$ melt temperature on the $40^{\circ} \mathrm{C}$ hot metal backing without any 


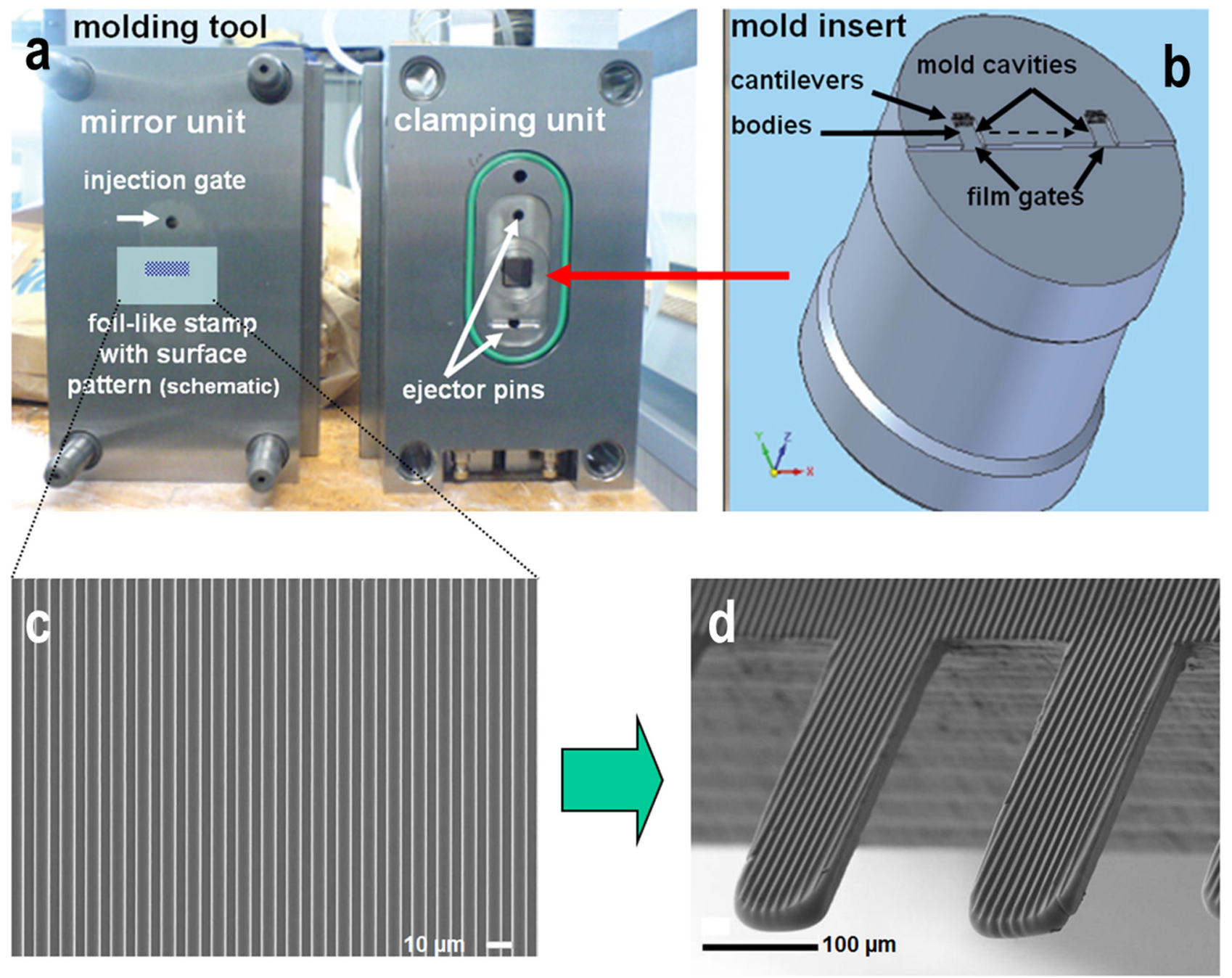

FIG. 4. (Color online) (a) Molding tool (handy mold) with two units (left side). The mirror side contains the gate (top) and the location of the patterned foil placement. (b) The clamping unit contains the mold insert with two mold cavities. The mold separation plane is between the polymer stamp on the mirror unit and the laser-machined insert on the clamping unit. (c) The imprinted PEEK foil and (d) its replication in PP $\mu$ Cs. Adapted with permission from Urwyler et al., Sens. Actuators A 172, 2 (2011); Figs. 1 and 6. Copyright 2011, Elsevier.

noticeable degradation. The actual temperature on the foil, however, was difficult to determine. While isothermal molding was used for the first generation of molds (see Figs. 3 and 4), the second generation enabled variothermal molding, with electric heaters placed just below the $\mu \mathrm{C}$ mold cavities. These heaters raised the temperature of the mold to above $T_{g}$ just before injection, and were simultaneously switched off to allow for cooling below $\mathrm{T}_{\mathrm{g}}$ after complete filling. This allowed for a lower mold temperature during injection and, therefore, a better and more defined cooling performance. However, as is a general characteristic of variothermal molding, longer process cycles of up to 1 min were needed.

\section{RESULTS AND DISCUSSION}

\section{A. Results}

\section{Micromechanical clamping elements with diffractive optical elements}

Similar to the micro-optical elements with single registration posts presented in Ref. 13, satisfactory molding parameters are also found for the micromechanical clamping elements. Due to the arrangement of the orifices around the microelement, distortion of the levers is avoided during demolding. While DOE surface patterns are clearly visible after $\mu \mathrm{IM}$ (see Fig. 3), the replication quality of the surface patterns does not meet that of the simpler micro-optical elements in Fig. 2. Furthermore, the alignment of the DOEs with respect to the microelement outlines is below a $1 \mu \mathrm{m}$ tolerance, which is insufficient for single-mode fiber coupling in an MT connector arrangement. Therefore, for these experiments, the DOE was not of a satisfactory quality to be optically relevant. While for the simpler structures, injection conditions may be found which enable good replication, the more complex larger structures still have insufficient quality and may need an optimized arrangement of injection nozzles and inlets.

\section{Microcantilevers with surface topographies}

Figures 5 and 6 show $\mu$ Cs with different surface patterns, which were manufactured using isothermal $\mu \mathrm{IM}$ of 

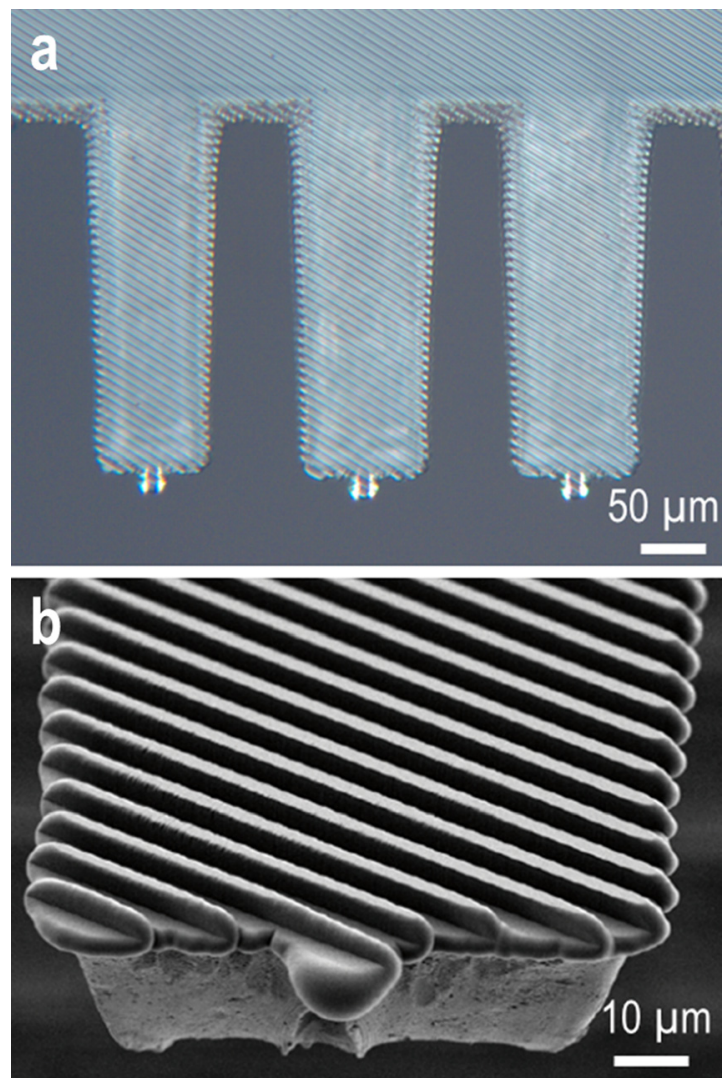

FIG. 5. (Color online) (a) Optical (top) and (b) SEM micrographs of completely molded microcantilevers with a diagonal line pattern (period $10 \mu \mathrm{m}$, depth $5 \mu \mathrm{m}$, and width $5 \mu \mathrm{m}$ ) transferred during an isothermal injection molding process from a foil-like mold insert to the surface (left side, size $\left.500 \times 80 \times 35 \mu \mathrm{m}^{3}\right)$. In contrast to the unpatterned original beams, the surface patterned beams are wider $(10 \%)$.

polypropylene. Compared to the vertical sidewalls of the micromechanical clamping elements, the $\mu \mathrm{Cs}(35-45 \mu \mathrm{m}$ thick and $500 \mu \mathrm{m}$ long) are easier to demold due to the draft angle of the mold cavities. Scanning electron microscope micrographs of the line pattern show that typical microstructures (period $10 \mu \mathrm{m}$, depth $5 \mu \mathrm{m}$, and width $5 \mu \mathrm{m}$ ) are successfully transferred from the foil mold insert to the surface of the polymeric $\mu \mathrm{Cs}$ during the molding process (see Fig. 5). In consequence, the surface area of the upper side of each $\mu \mathrm{C}$ is doubled. In contrast to the unpatterned original beams, the surface patterned beams are slightly wider $(10 \%)$ at the upper side. This widening is always present in completely molded $\mu \mathrm{Cs}$, but it is enhanced when the gratings are perpendicular or diagonal to the $\mu \mathrm{C}$ beams. When this is the case, the line cavities enable the melt to flow in the transverse direction and protrude along the small grating cavities in the areas where the unstructured mold area presses onto the foil, forming small areas of extra material, which can be considered "flash." While this is a clear sign that the molding pressure is sufficiently high up to the end of the $\mu \mathrm{C}$ beams (see the $\mu \mathrm{C}$ tips where the melt begins to fill the venting channels), nanostructures are more difficult to mold. However, in Fig. 6, we demonstrate that protruding square pyramids with a $2 \mu \mathrm{m}$ footprint can be replicated and tips with an apex of about $100 \mathrm{~nm}$ can be replicated as well (see
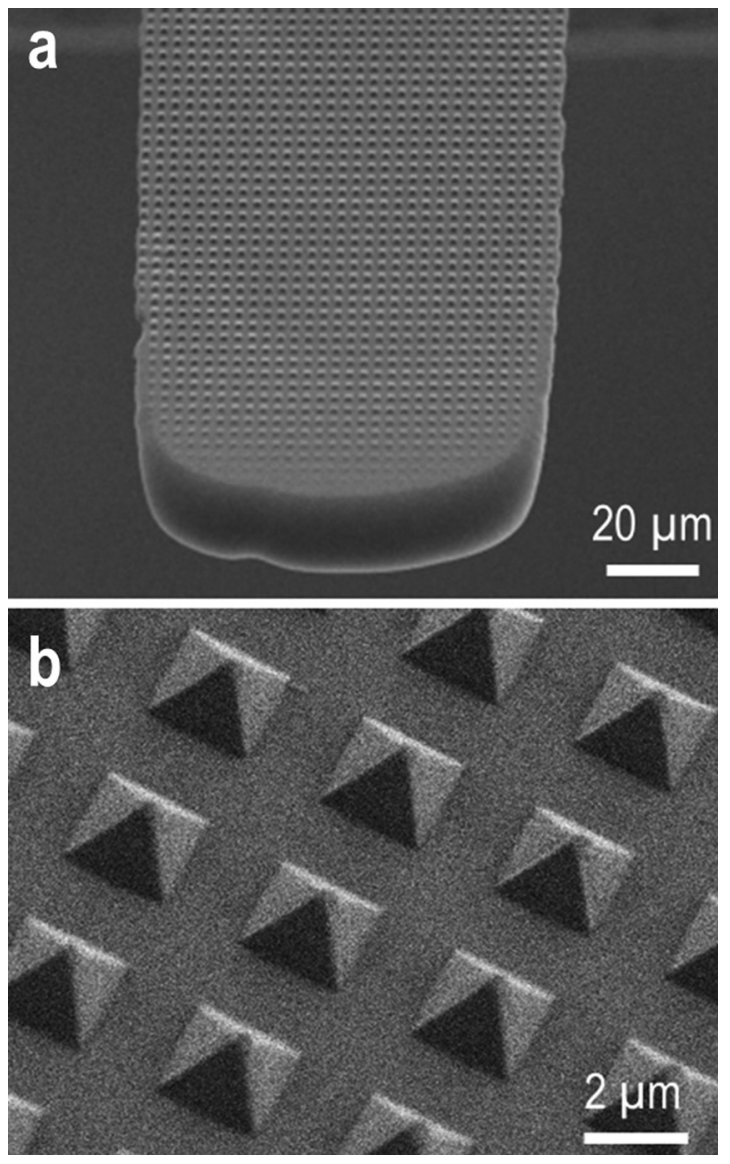

FIG. 6. (a) SEM micrographs showing microcantilever with pyramid patterns (period $4 \mu \mathrm{m}$, footprint $2 \mu \mathrm{m}$, and height $1.4 \mu \mathrm{m}$ ) transferred during the molding process from a foil-like mold to the cantilever surface in an isothermal injection molding process. (b) Although the cavity is not completely filled, the pyramids are molded almost up to the tip of the cantilever.

Fig. 6). Although the cavity was not completely filled, the pyramids are almost completely molded up to the tip of the cantilever. This proves that surface patterns with submicrometer resolution can be molded even in the area where the $\mu \mathrm{C}$ cavity is most sensitive. However, due to insufficient alignment capabilities, no single pyramidal tip could be accurately placed. Further results show that during separation the structures are prone to distortion.

\section{B. Discussion}

Both mold configurations show different challenges during molding and demolding, and both have their specific advantages. For the micromechanical clamping elements, levers with an aspect ratio of 5 and with $500 \mu \mathrm{m}$-high vertical sidewalls can be molded using a variothermal $\mu \mathrm{IM}$ process. While the molding of the levers is possible by using a configuration of orifices for melt injection around the microelement, it was not sufficient to replicate the surface of the DOE topography. However, since these experiments were done before the molding of the $\mu \mathrm{Cs}$ presented here, it is possible that better DOE replication can be achieved if polymer inlays are used instead of the metal shims. The assembly of two metal molds in a combined mold enables the prealignment of two molds with respect to each other, which is a 
prerequisite if submicrometer precision is required. Due to the limited ability to preserve the alignment achieved in an optical microscope during clamping and fixation, this method can only lead to good results if specialized equipment is used or redundant trials are pursued until satisfactory alignment is achieved. Once the mold is inserted, it yields good results. However, the $\mu \mathrm{IM}$ conditions need to be optimized to allow good replication of both frames and surface patterns, possibly by including larger or more inlets. For this purpose, Moldflow simulations can be quite useful, as evidenced by the work on $\mu \mathrm{Cs} .{ }^{18}$

The use of a modular mold configuration to create surface patterned $\mu \mathrm{Cs}$ resulted in astonishing results. Particularly, it enabled us to combine the low thermal conductivity and the patterning of a thin PEEK foil used as the "mirror side" of the $\mu \mathrm{C}$ cavity. It is far from proven that the foil will survive more than 50 injection cycles without severe degradation. However, the current results suggest that for prototyping or small-scale production the method of polymeric inlays can be applied successfully. Since the foil patterns are fabricated using hot embossing, a feeder mechanism could be used to provide new foils once they begin to degrade, similar to what is being done in conventional in-mold labeling. The current application may support such a setup, since it was not designed for aligned structures. The widening of structures, however, must be inhibited or controlled by using variothermal molding schemes or specific structures, which reduce the generation of localized flash. In this work, the formation of flash may be facilitated by the high injection pressure and the softness of the PEEK foil. Also, fixing the foil with Scotch tape may be inadequate for experiments requiring higher reliability. Nevertheless, these results show that the surface topography are filled almost to the end of the patterns and suggest that further improvements can be achieved by careful optimization of tool design (particularly, hybrid mold inlay fixation) and process parameters (particularly, the use of variothermal $\mu \mathrm{IM}$ ). Instead of using PEEK, crosslinked polymers such as SU-8 may enable further improvement of the mold durability and even allow the fabrication of mold inserts with precise alignment. ${ }^{19}$ In this way, polymer inlays may be considered for more complex geometries such as micromechanical clamping elements.

\section{SUMMARY AND CONCLUSIONS}

The main finding of this research is that both hybrid mold configurations work well in principle, but with severe restrictions in molding fidelity or dimensional tolerances. Future research will enhance knowledge and develop variants, which can provide better alignment and high replication fidelity. In any case, the hybrid mold insert approach will enable increased flexibility for prototyping and may even find industrial applications in certain niches. For more precise manufacturing, hybrid methods need to be applied to fabricate monolithic molds with both micro- and nanostructures in the same mold, e.g., by mixing and matching different methods. ${ }^{20}$ However, the specific advantage of surface patterns could be compromised if nanostructures are not placed on the top or the bottom of a $21 / 2 \mathrm{D}$ or $3 \mathrm{D}$ structure, and if they are allowed to interfere with molding of the microelements. For instance, when high aspect ratio pillars cover areas of the mold, which does not enable demolding along a defined axis, distortions and even rupture are inevitable. ${ }^{21}$ Examples are polymer parts (e.g., LEGO bricks) with integrated photonic surface structures (structural colors) ${ }^{22}$ or microfluidic channels with small high aspect ratio micro- or nanopillars for fluid control. The latter application is envisaged for the purpose of rendering areas of the surface hydrophobic (instead of applying hydrophobic chemical coatings) and has already been used in fuel cells where liquid inputs and gaseous outputs must be separated using localized antiwetting properties. ${ }^{23}$ Furthermore, the overall increase of the surface area due to topographical structuring may lead to increased demolding forces, which may make replication more delicate.

\section{ACKNOWLEDGMENTS}

The authors thank several people for their help and valuable contributions. The LiGA structures were manufactured at the Institute for Microtechnology Mainz (IMM GmbH) with the help of N. Zimmerschitt, R. Lüttge, and M. Schmidt. Diffractive optical devices were fabricated with the help of R. Stutz and J. Söchtig at the former Paul Scherrer Institut, Zürich. The polymer microcantilevers were injection molded at the University of Applied Sciences and Arts Northwestern Switzerland (FHNW) with the help of O. Häfeli. The $\mu \mathrm{C}$ molds were fabricated with picosecond pulsed laser ablation by B. Lüscher at FHNW and K. Jefimovs at EMPA Dübendorf. Hybrid polymer mold inlays were hot embossed by M. Altana, K. Vogelsang, and C. Spreu at PSI. The research presented here was partially funded by the 7th framework program of the European Commission (project NaPANIL) and the Swiss Nanoscience Institute (project DICANS).

${ }^{1}$ M. J. Madou, Fundamentals of Microfabrication: The Science of Miniaturization, 2nd ed. (CRC, Boca Raton, FL, 2002).

${ }^{2}$ H. P. Lang, M. Hegner, and Ch. Gerber, "Nanomechanical cantilever array sensors," in Handbook of Nanotechnology, edited by B. Bhushan (Springer, Berlin, 2010), pp. 427-452.

${ }^{3}$ W. Menz, J. Mohr, and O. Paul, Microsystem Technology (John Wiley \& Sons, Weinheim, 2008)

${ }^{4}$ V. Saile and U. Wallrabe, "LIGA and its applications," in Advances in Micro and Nanosystems (John Wiley \& Sons, Weinheim, 2009), Vol. 7, pp. 351-394.

${ }^{5}$ M. Calleja, J. Tamayo, L. M. Lechuga, and A. Boisen, Ultramicroscopy $\mathbf{1 0 5}, 215$ (2005)

${ }^{6}$ S. A. Mimotec, see: http://www.mimotec.ch/

${ }^{7}$ J. Köser, J. Gobrecht, U. Pieles, and B. Müller, Eur. Cells Mater. 16, 38 (2008).

${ }^{8}$ M. A. F. van den Boogaart, L. M. Doeswijk, and J. Brugger, J. Microelectromech. Syst. 15, 1663 (2006).

${ }^{9}$ H. Schift, A. D’Amore, C. David, M. Gabriel, J. Gobrecht, W. Kaiser, and D. Simoneta, J. Vac. Sci. Technol. B 18, 3564 (2000).

${ }^{10}$ J. Giboz, T. Copponnex, and P. Mélé, J. Micromech. Microeng. 17, 96 (2007).

${ }^{11}$ M. A. P. Andrew, W. McFarland, L. A. Bottomley, and J. S. Colton, Nanotechnology 15, 1628 (2004).

${ }^{12}$ J. Söchtig, H. Schift, P. D. Patterson, and S. Westenhöfer, Proc. SPIE 3226, 44 (1997).

${ }^{13}$ H. Schift, J. Söchtig, M. Rossi, and S. Westenhöfer, Proc. SPIE 3513, 122 (1998). 
${ }^{14}$ P. Urwyler, H. Schift, J. Gobrecht, O. Häfeli, M. Altana, F. Battiston, and B. Müller, Sens. Actuators A 172, 2 (2011).

${ }^{15}$ P. Urwyler, J. Köser, H. Schift, J. Gobrecht, and B. Müller, Biointerfaces 7, 6 (2012).

${ }^{16}$ H. Pranov, H. K. Rasmussen, N. B. Larsen, and N. Gadegaard, Polym. Eng. Sci. 46, 160 (2006).

${ }^{17}$ M. Matschuk and N. B. Larsen, J. Micromech. Microeng. 23, 025003 (2013).

${ }^{18} \mathrm{P}$. Urwyler, "Polymeric micro-cantilever sensors for biomedical applications," Ph.D. dissertation (University of Basel, Switzerland, 2012).
${ }^{19}$ J. M. Stormonth-Darling and N. Gadegaard, Macromol. Mater. Eng. 297, 1075 (2012).

${ }^{20}$ A. Schleunitz, C. Spreu, J. J. Lee, and H. Schift, J. Vac. Sci. Technol. B 28, C6M41 (2010).

${ }^{21}$ T. Senn, Ch. Waberski, J. Wolf, J. P. Esquivel, N. Sabaté, and B. Löchel, Microelectron. Eng. 88, 11 (2011).

${ }^{22}$ J. Clausen, A. B. Christiansen, J. Garnaes, N. A. Mortensen, and A. Kristensen, Opt. Express 20, 4376 (2012).

${ }^{23}$ J. P. Esquivel, T. Senn, P. Hernandez-Fernandez, J. Santander, M. Lörgen, S. Rojas, B. Löchel, C. Cané, and N. Sabaté, J. Power Sources 195, 8110 (2010). 